\title{
Addiction peer recovery coach training pilot: Assessment of confidence levels
}

\author{
Nicholas Guenzel ${ }^{\text {Corresp., } 1}$, Hongying Dai ${ }^{2}$ \\ 1 University of Nebraska Medical Center, Lincoln, Nebraska, United States \\ 2 University of Nebraska Medical Center, Omaha, Nebraska, United States \\ Corresponding Author: Nicholas Guenzel \\ Email address: nguenzel@unmc.edu
}

Background: Peer recovery coaches (PRCs) have become a critical tool in addiction treatment in many areas of the world. Despite this fact, no identified research has examined the process or impact of PRC training. Furthermore, no scales were identified to measure trainee confidence in various PRC techniques. The goal of this paper is to analyze the process and immediate impact of PRC training of twelve American Indians (Als) in a culturally-specific program. We focus most specifically on trainee confidence levels. Methods: No written consent was obtained and completion of the assessment was considered consent. Trainees completed self-assessments before and after the training. The self-assessment examined nine areas ranging from understanding the role of PRCs to knowledge of effective PRC techniques. Paired t-tests were used to assess for changes in individual trainee responses between the pre- and post-assessments. Results: Pre-training responses ranged from moderate to high. Questions with the lowest average confidence levels address PRC activities or specific techniques to facilitate recovery. All nine questions showed statistically significant mean improvements in the post-training self-assessments. Questions regarding specific PRC activities and techniques showed the greatest improvement. Questions relating to helping people more generally showed the smallest improvement. Average post-training responses fell within a very narrow range indicating relatively consistent confidence levels across skills. Analysis indicates participants were possibly over-confident in certain areas (i.e. maintaining boundaries). This small pilot represents an initial attempt to measure confidence levels of PRC trainees. The findings may inform future training by identifying certain areas where emphasis might be most helpful for trainees. In addition, it is hoped that this work will encourage more systematic analysis of the impact of PRC training on individuals. 
1 Addiction peer recovery coach training pilot: Assessment of confidence levels

2

3

4 Nicholas Arien Guenzel ${ }^{1}$, Hongying Dai ${ }^{2}$

5

$6{ }^{1}$ College of Nursing, University of Nebraska Medical Center, Lincoln, Nebraska, USA

$7 \quad{ }^{2}$ College of Public Health, University of Nebraska Medical Center, Omaha, Nebraska, USA

8

9 Corresponding Author:

10 Nicholas Guenzel $^{1}$

$11550 \mathrm{~N} 19^{\text {th }}$ Street, Lincoln, Nebraska, 68588-0620, USA

12 Email address: nguenzel@unmc.edu

13 
14 Abstract

15 Background: Peer recovery coaches (PRCs) have become a critical tool in addiction treatment in

16 many areas of the world. Despite this fact, no identified research has examined the process or

17 impact of PRC training. Furthermore, no scales were identified to measure trainee confidence in

18 various PRC techniques. The goal of this paper is to analyze the process and immediate impact of

19 PRC training of twelve American Indians (AIs) in a culturally-specific program. We focus most

20 specifically on trainee confidence levels.

21 Methods: No written consent was obtained and completion of the assessment was considered

22 consent. Trainees completed self-assessments before and after the training. The self-assessment

23 examined nine areas ranging from understanding the role of PRCs to knowledge of effective PRC

24 techniques. Paired t-tests were used to assess for changes in individual trainee responses between

25 the pre- and post-assessments.

26 Results: Pre-training responses ranged from moderate to high. Questions with the lowest average

27 confidence levels address PRC activities or specific techniques to facilitate recovery. All nine

28 questions showed statistically significant mean improvements in the post-training self-

29 assessments. Questions regarding specific PRC activities and techniques showed the greatest

30 improvement. Questions relating to helping people more generally showed the smallest

31 improvement. Average post-training responses fell within a very narrow range indicating

32 relatively consistent confidence levels across skills. Analysis indicates participants were possibly

33 over-confident in certain areas (i.e. maintaining boundaries). This small pilot represents an initial

34 attempt to measure confidence levels of PRC trainees. The findings may inform future training by

35 identifying certain areas where emphasis might be most helpful for trainees. In addition, it is

36 hoped that this work will encourage more systematic analysis of the impact of PRC training on

37 individuals. 
Introduction

40

Individuals seeking recovery from addiction have doubtlessly been using peer recovery

41 for centuries. Peer recovery groups such as Alcoholics Anonymous were formalized in the early

$4220^{\text {th }}$ century (Kurtz, 2010). Specific volunteer and paid roles for peer workers have been

43 established in various ways since the 1840s (White, 2010). However, early roles almost certainly

44 lacked the preparation and standards seen in peer recovery coach (PRC) certifications of today.

45 - Some authors have conceptualized PRC services as a bridge between professional addiction

46 treatment and recovery mutual aid (White, 2010). Research has identified that PRCs connect

47 with the people they serve based on "trust and shared lived experiences" rather than the

48 professional training of healthcare providers (Collins et al., 2019, p. 6). Although peer recovery

49 coaches hold great promise, limited research has examined their training. The purpose of this

50 article is to assess a peer recovery coach training program for American Indians (AIs) that was

51 conducted in the fall of 2019.

52 In contrast to many other interventions to address health problems, it seems intuitive for

53 individuals who have succeeded in the process of recovery to guide others seeking the same. In

54 part, it appears this natural process has led to great diversity in peer recovery services. Various

55 titles including peer recovery coaches, peer recovery specialists, peer workers, peer navigators,

56 peer mentors, wellness coaches and others have been used for individuals providing support.

57 Although the use of PRCs has grown internationally, the specific roles of support individuals

58 have also varied greatly (White, 2010). In some cases, the services delivered by individuals with

59 these titles may be quite similar but in others they may be very diverse. Without standardized

60 terms and defined roles, it is difficult to compare the services of support individuals across

61 programs.

62 Interventions have ranged in duration from as short as one meeting with a telephone

63 follow-up to long-term relationships over the course of months or years. Many programs have

Peer) reviewing PDF | (2020:09:52703:1:0:NEW 7 Dec 2020) 
64 targeted specific populations including individuals with co-occurring mental health problems,

65 pregnant women, individuals in the criminal justice system, people living in supported housing,

66 and people with HIV (Reif et al., 2014). Some researchers have found that the use of PRCs has

67 been associated with reduced substance use and increased medical service engagement (Cos et

68 al., 2019). However, the heterogeneity of PRC studies has limited the ability of researchers to use

69 metanalyses to draw clear conclusions (Eddie et al., 2019). More standardized training and

70 assessment could play a role in improving consistency across studies and projects (Elsinger \&

71 Rentsch, 2019). Only recently have researchers started to examine the needs of PRCs including

72 the challenges of vulnerability, authenticity, boundaries, stigma, and lack of recognition (Miler et

73 al., 2020). To further work towards more consistent trainee assessment, the first author developed

74 a self-assessment scale measuring trainee confidence in a variety of PRC tasks.

75 Searches of the literature did not locate PRC treatment programs specific to any cultural

76 groups other than AIs (Kelley et al., 2017). Many AI cultures may be particularly well suited for

77 PRC work for several reasons. First, AIs have a long history of "recovery circles" where

78 members in recovery support each others' efforts, often using AI traditions such as sweat lodges

79 and talking circles (White, 2010). Second, unlike many European cultures, intoxicating

80 substances appear to have played a very minimal role in AI groups outside of ceremonial use

81 (Frank, Moore \& Ames, 2000). Third, the AI values of respect for traditional ways and a more

82 collective orientation may lead AIs to be more receptive to PRC interventions (Rowan et al.,

83 2014).

84 Most states have established a PRC certification process. No national process has been

85 established for PRC certification and no standard competencies have been proposed. The number

86 of hours of specific PRC education varies by state but are commonly between 60 and 100 hours.

87 Most states require paid or volunteer experience which varies greatly between 100 and 1,000

88 hours (Blash, Chan \& Chapman, 2015). In addition, many certifications require a defined number 
89 of hours of supervised work experience that can vary greatly between 24 and 500 hours. Lastly,

90 aspiring PRCs are required to pass a certification exam in most states (Myrick \& del Vecchio,

91 2016). The broad requirements for certification make it clear that a great amount of diversity

92 exists in PRC preparation. No articles reviewing the PRC training process were located so this

93 work will seek to examine the ways in which one training program prepared PRCs.

94 The training examined in this paper was part of a study to assess the impact of AI PRCs in

95 assisting other AIs in the process of recovery from alcohol addiction. The project is being carried

96 out in collaboration with the Nebraska Urban Indian Health Coalition, the Society of Care, and

97 the Southeast Nebraska Native American Collation. The training and study incorporate AI values

98 and traditions which include a cultural history of sobriety, spiritual implications for intoxication,

99 and greater consciousness of individual actions on the collective community. In addition, the

100 training and coaching makes use of AI spiritual practices (i.e. sweat lodges, talking circles) which

101 require and can facilitate sobriety. In the understanding of the authors, the AI aspects of the

102 training and coaching give individual coaches more tools to use with individuals in recovery.

103 However, in most cases they do not fundamentally change the role of the coach.

104 The first author of this article contracted with two trainers from Michigan to provide a 20-

105 hour PRC training program. Six AIs from Lincoln and six AIs from Omaha completed the

106 training over three days in a Nebraska state park. The trainees include four men and eight women

107 ranging in age from 33 to 61.

Aims

109 1. Examine the absolute confidence levels in the nine areas addressed by the pre-training

110 self-assessment tool and the relative confidence levels between the areas

111 2. Examine the change scores between the pre- and post-training assessments

112 3. Examine correlations between the change scores of the questions

\section{Methods}


114 Trainees were identified through the primary investigator's contacts from previous projects, the

115 Nebraska Urban Indian Health Coalition, the Society of Care, and the Southeast Nebraska Native

116 American Coalition. Please see Figure 1 for a flowchart of the project.

117 Trainers used several methods to deliver content. Some information was relayed in the form of

118 presentations. Discussions were also held to facilitate understanding. Numerous videos were

119 shown including autobiographical stories in which AIs reviewed their recovery journeys. The

120 most interactive portion of the training consisted of numerous role-playing activities in which one

121 trainee acted as the PRC and the other as the individual in recovery. The role-playing allowed

122 trainees to practice and demonstrate what they had learned. These exercises also triggered

123 extensive discussion about a wide variety of topics relating to the work of PRCs and addiction

124 more generally.

125 The program was opened with an elder performing prayers and smudging. The trainers

126 then proceeded with an overview of the program followed by example descriptions of what AI

127 PRCs do in their work. During this time, each participant was asked to describe their background

128 and what brought them to this training. The Anishnaabek Healing Circle was then examined as a

129 model for tribal/urban PRC programs.(Inter-Tribal Council of Michigan, 2020). The groups were

130 then divided between people from Omaha and those from Lincoln. The two groups were asked to

131 identify the legal, cultural, physical, spiritual, and mental resources in each city. Copies of these

132 lists were then distributed to the trainees for use in assisting the people with whom they work.

133 The next section of the training gave an overview of the structure and function recovery

134 management plans. Trainers then discussed the process of developing recovery management

135 plans.

136 During the next section, the trainers went into greater detail regarding the roles and

137 responsibilities of a PRC. Specifically, discussion of how PRCs differ from counselors, sponsors,

138 and others helped trainees understand how PRCs operate. Strategies for managing professional 
139 boundaries were also discussed including ethics, confidentiality, power differentials, and

140 challenges during crisis management. The trainers then discussed the support available for PRCs

141 and how PRCs can do their part in maintaining effective relationships with their supervisors.

142 On the final day, the trainers reviewed the additional steps the trainees would need to

143 complete to become certified. This included strategies self-study using the Rhode Island Exam

144 Study Guide, the examination, required work hours, and required supervised PRC activities

145 (Department of Behavioral Healthcare Developmental Disabilities \& Hospitals, 2016; Nebraska

146 Department of Health and Human Services, 2020). The next part of the day was spent exploring

147 and reinforcing skills learned during the previous two days through various role-playing

148 activities. The training concluded with a large group sharing exercise where everyone discussed

149 their thoughts and feelings as they progressed through the training.

150 The first author developed nine questions based on the specific curriculum of the training

151 to assist in assessing the trainees' confidence in PRC activities (see Table 1). Trainees could

152 circle any number from 1 ("disagree") to 9 ("agree") to indicate their response to the question.

153 Paper copies of the questions were given to participants before the training started. The same

154 procedure was repeated at the end of the training. The authors then entered the pre- and post-

155 responses for each individual into SPSS. Paired t-tests were then run to determine if trainee

156 responses changed after the training. Pearson correlations were calculated to measure the

157 association of change in confidence level between each pair of questions. Statistical analyses

158 were performed using SAS 9.4 (Cary, NC) and statistical significance was claimed with p-value $159<0.05$.

\section{Results}

163 Aim 1. 

assessment with means ranging from 4.7 to 7.4 (see Table 1). In five of the seven questions, the mean pre-response was seven or above. Only two questions had mean pre-responses of 5.4 or

167 lower reflecting a moderate level of confidence. However, it is worth noting that the three

168 questions with the lowest level of initial agreement specifically addressed PRC roles and

169 activities. The other question that rated somewhat lower addressed specific techniques to

170 facilitate recovery. All other questions had an average initial response of 7 or higher.

171 Aim 2.

Despite the moderate to high level of initial confidence trainees had in response to most

173 questions and the small sample size, statistically-significant increases in their confidence were

174 found in responses to all ten questions at the $\mathrm{p}<0.05$ level. The standard deviations were also

175 smaller in the post-training assessment relative to the pre-training assessment. As might have

176 been predicted, the largest mean difference between the two surveys specifically asked about

177 aspects of PRCs (understanding the role, defining the role and responsibilities, confidence in

178 serving as an effective PRC, and having effective techniques to help individuals in recovery) and

179 also had the lowest initial ratings. The five questions with the smallest changes (1.25 to 1.58)

180 related to more general aspects of recovery support rather than PRC-specific skills and tasks.

181 These included being able to help, feeling self-assured in helping, maintaining boundaries,

182 feeling comfortable in assisting people, and helping develop a recovery plan.

183 Aim 3.

184 Pearson correlations were calculated to measure the association of change between 185 questions (see Table 2). The negative sign stands for the inverse association while the magnitude 186 of correlation measures the strength of the association. Many strong associations were noted

187 between the questions. The strongest correlations both had a coefficient of 0.88 . The first was 188 between "understanding the role of a PRC" and "defining the roles and responsibilities of a 
189 PRC." The second was between "feeling self-assured in helping people in recovery" and "having 190 effective techniques for helping individuals in recovery."

191

192 Discussion

193 Although PRCs are widely employed in helping individuals recover from addiction, very

194 little research has examined the effect of their support and no identified research has focused on

195 their training. To our knowledge, this paper represents the first analysis of initial confidence

196 levels of PRC trainees in specific PRC-related areas and the changes that occurred as a result of

197 the training. The authors contend that systematic analysis of the impact of training methods is

198 essential for the most effective preparation of peer recovery coaches.

199 A number of previously-undiscussed issues became apparent during the analysis of the

200 self-assessments. For example, it appears likely that trainees were over-confident regarding their

201 ability to maintain professional boundaries as a PRC. This was reflected during the training

202 where trainees made a number of statements reflecting their previous lack of understanding. The

203 initial mean score of 7.08 is especially high for two reasons. First, as indicated by the three

204 questions addressing aspects of PRC work which were scored the lowest in the pre-assessment,

205 trainees admitted to having low information on PRCs relative to more general aspects of

206 recovery. It seems unlikely that individuals who have a limited understanding of PRC roles and

207 responsibilities would be highly skilled in maintaining boundaries which differ significantly from

208 other roles they may have experienced. Second, the question of being skilled at maintaining

209 boundaries necessitates that they not only know what boundaries exist but having techniques

210 needed to enforce them in challenging situations. The responses to the boundaries question and

211 the questions relating to PRCs specifically indicate that trainers need to focus a significant

212 amount of time on defining PRC boundaries. PRCs have roles that are quite different from 
213 sponsors, therapists, and others with which trainees may be familiar. Exercises such as role-

214 playing can help trainees practice asserting these boundaries.

215 Another significant point in this analysis is that there was little change in scores reported

216 that regarded more general aspects of helping individuals in recovery. The most prominent

217 examples included the questions of being able to help people in recovery and feeling self-assured

218 in helping people. It appears that trainees came into the program already having a significant

219 amount of confidence in their ability to assist people in the process of recovery. This confidence

220 is likely beneficial as individuals who believe they can help others will likely be more willing to

221 engage with individuals in recovery than those who are less confident. However, we must note

222 that confidence may not equate to competence. As a result, general principles of assisting

223 individuals in recovery are still a critical part of PRC training.

224 The last significant point to note is that the greatest increase in confidence was seen in the 225 three questions specifically related to PRC roles. It is logical that these three questions had the

226 lowest initial scores as aspects of PRC work are specific to that role rather than more general 227 principles of recovery support. Individual experiences in treatment, support groups, or other

228 settings foster general recovery knowledge but would be unlikely to give individual insight into

229 PRC work. The low initial scores clearly provided the most room for increased confidence.

230 However, the fact that the post- scores were within a very narrow range with the rest of the

231 questions indicate that the training was effective in at least increasing the confidence of trainees

232 in PRC roles and responsibilities.

233 This analysis had a number of limitations. First, the analysis is based on a small sample

234 size due, in part, to the fact that such intimate training sessions commonly involve a small

235 number of trainees. Multiple cohorts would likely be needed to draw more reliable conclusions.

236 Second, since the assessments were completed for the training rather than research, we did not

237 have access to more detailed demographic information for each trainee. The training involved a 
238 number of AI values and traditions but the assessment questions did not focus on these

239 specifically so it appears likely that similar patterns may be found in more general populations.

\section{Conclusions}

242 The lack of a national certification process or universally-accepted standards should not

243 be a barrier to establishing program were PRCs can help individuals in the process of recovery.

244 This analysis has demonstrated that training can significantly improve the confidence of future

245 PRCs in a wide variety of areas. However, due to the limitations of this analysis, further study

246 and replication is warranted. In particular, more extensive training might start with trainees'

247 baseline level of knowledge which may give insight into confidence and potential

248 misunderstandings in certain areas.

249 PRCs commonly bring valuable experience of addiction but often lack an understanding 250 of the more intricate roles, responsibilities, and techniques of a PRC. Training helps individuals 251 transform this vast experience into tools that will assist others in the recovery from addiction. 


\section{References}

254

255 Blash L, Chan K, Chapman S. 2015. The peer provider workforce in behavioral health: A landscape analysis. San Francisco, CA: UCSF Health Workforce Research Center on

258 Collins D, Alla J, Nicolaidis C, Gregg J, Gullickson DJ, Patten A, Englander H. 2019. “If It Wasn't for Him, I Wouldn't Have Talked to Them": Qualitative Study of Addiction Peer Mentorship in the Hospital. Journal of general internal medicine:1-8.

Cos TA, LaPollo AB, Aussendorf M, Williams JM, Malayter K, Festinger DS. 2019. Do Peer Recovery Specialists Improve Outcomes for Individuals with Substance Use Disorder in

Department of Behavioral Healthcare Developmental Disabilities \& Hospitals. 2016. Rhode an Integrative Primary Care Setting? A Program Evaluation. Journal of Clinical Island Peer Recovery Specialist Certification. Available at https://docs. Psychology in Medical Settings:1-12. wixstatic.com/ugd/4208dd_b583e05c31d448deafda8b9b2d781927.pdf(accessed December 1, 2020).

Eddie D, Hoffman L, Vilsaint C, Abry A, Bergman B, Hoeppner B, Weinstein C, Kelly JF. 2019. Lived experience in new models of care for substance use disorder: A systematic review of peer recovery support services and recovery coaching. Frontiers in psychology 10:1052.

Elsinger J, Rentsch J. 2019. Fighting Opioid Addiction in the United States: Legislative Policy Review, and How Peer Supports are Suited for Battle. The Graduate Review 4:8-22. Frank JW, Moore RS, Ames GM. 2000. Historical and cultural roots of drinking problems among American Indians. American Journal of Public Health 90:344-351. 
277 Inter-Tribal Council of Michigan. 2020.The Anishnaabek Healing Circle. Available at 278 https://www.atrhealingcircle.com/

279 Kelley A, Bingham D, Brown E, Pepion L. 2017. Assessing the Impact of American Indian Peer 280 Recovery Support on Substance Use and Health. Journal of Groups in Addiction \& $281 \quad$ Recovery 12:296-308.

282 Kurtz E. 2010. Not god: A history of Alcoholics Anonymous. Simon and Schuster.

283 Miler JA, Carver H, Foster R, Parkes T. 2020. Provision of peer support at the intersection of 284 homelessness and problem substance use services: a systematic 'state of the art'review. 285 BMC public health 20:1-18.

286 Myrick K, del Vecchio P. 2016. Peer support services in the behavioral healthcare workforce: State of the field. Psychiatric rehabilitation journal 39:197.

288 Nebraska Department of Health and Human Services. 2020.Peer Support Specialist Core 289 Curriculum and Certification Guidelines. Available at http://dhhs.ne.gov/Pages/Peer290 Support-Training-Certification.aspx (accessed December 1, 2020).

Reif S, Braude L, Lyman DR, Dougherty RH, Daniels AS, Ghose SS, Salim O, Delphin-Rittmon 292 ME. 2014. Peer recovery support for individuals with substance use disorders: Assessing the evidence. Psychiatric Services 65:853-861.

Rowan M, Poole N, Shea B, Gone JP, Mykota D, Farag M, Hopkins C, Hall L, Mushquash C, Dell C. 2014. Cultural interventions to treat addictions in Indigenous populations: findings from a scoping study. Substance abuse treatment, prevention, and policy 9:34.

297 White WL. 2010. Nonclinical addiction recovery support services: History, rationale, models, 298 potentials, and pitfalls. Alcoholism Treatment Quarterly 28:256-272. 299 
Figure 1

Project flow chart 
Figure 1. Project Flow Chart

Trainee recruitment from community

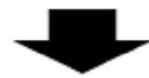

In-person training arranged

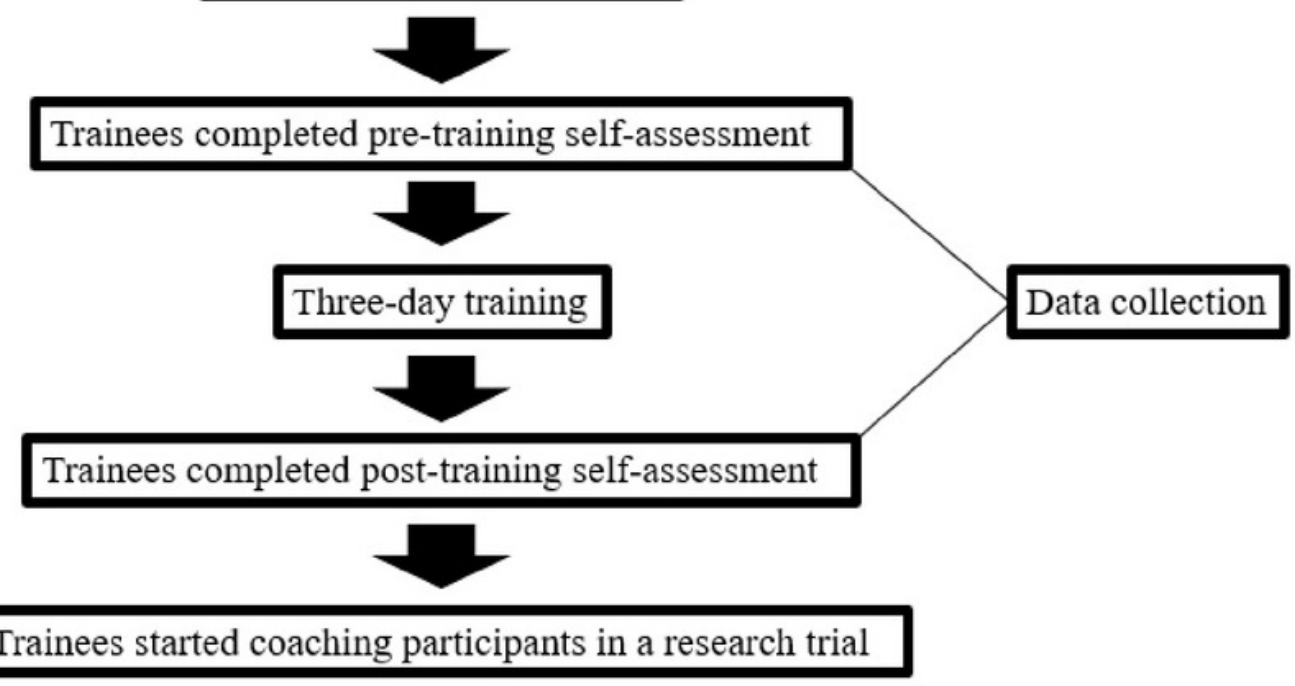




\section{Table 1 (on next page)}

Pre- and Post-Training Confidence Assessment Questions 
Table 1. Pre- and Post-Training Confidence Assessment Questions ${ }^{\mathrm{a}}$

\begin{tabular}{|l|l|l|l|l|}
\hline & $\begin{array}{l}\text { Pre: mean } \\
(\mathrm{SD})\end{array}$ & $\begin{array}{l}\text { Post: mean } \\
(\mathrm{SD})\end{array}$ & $\begin{array}{l}\text { Mean change } \\
(\mathrm{SD})\end{array}$ & $\mathrm{P}^{\text {P-value }}$ \\
\hline 1. I understand the role of a peer recovery coach & $5.4(2.9)$ & $8.7(0.7)$ & $3.3(2.7)$ & $0.0016^{* *}$ \\
\hline 2. I am comfortable in assisting people in an early stage of recovery & $7.3(1.9)$ & $8.8(0.5)$ & $1.5(1.7)$ & $0.0121^{*}$ \\
\hline 3. I can define the roles and responsibilities of a peer recovery coach & $4.7(3.0)$ & $8.6(0.8)$ & $3.9(3.0)$ & $0.0008^{* *}$ \\
& & & & $0.0404^{*}$ \\
\hline 4. I am able to help individuals in recovery from addiction & $7.3(2.0)$ & $8.6(0.8)$ & $1.3(1.9)$ & $0.0033^{* *}$ \\
\hline $\begin{array}{l}\text { 5. I am skilled at maintaining professional boundaries in challenging } \\
\text { situations }\end{array}$ & $7.1(1.7)$ & $8.5(0.8)$ & $1.4(1.3)$ & $0.0112^{*}$ \\
\hline 6. I feel self-assured in helping people in recovery & $7.4(1.6)$ & $8.7(0.5)$ & $1.3(1.4)$ & $0.0122^{*}$ \\
\hline 7. I would do well in helping someone develop a recovery plan & $7.0(2.1)$ & $8.6(0.7)$ & $1.6(1.8)$ & $0.0045^{* *}$ \\
\hline 8. I am able to serve as an effective peer recovery coach & $6.3(2.4)$ & $8.7(0.7)$ & $2.3(2.3)$ & $0.0064^{* *}$ \\
\hline 9. I have effective techniques for helping individuals in recovery & $6.3(2.5)$ & $8.4(0.8)$ & $2.1(2.2)$ & \\
\hline
\end{tabular}

${ }^{a}$ : Each question was rated by a scale from 1 (disagree) to 9 (agree).

${ }^{b}$ : Paired t-test was performed to compare confidence level before and after training.

$* \mathrm{p}<0.05$

$* * \mathrm{p}<0.01$

$* * * \mathrm{p}<0.001$ 
Table 2 (on next page)

Pearson Correlation for the Change of Confidence Levels 
Table 2. Pearson Correlation for the Change of Confidence Levels ${ }^{\mathrm{a}}$

\begin{tabular}{|c|c|c|c|c|c|c|c|c|}
\hline & Assisting & Define & Help & Boundaries & $\begin{array}{c}\text { Self- } \\
\text { assured }\end{array}$ & $\begin{array}{c}\text { Recovery } \\
\text { plan }\end{array}$ & Effective & Techniques \\
\hline Role & $0.15(0.65)$ & $\begin{array}{c}0.88 \\
(0.0002)^{* * *}\end{array}$ & $\begin{array}{c}0.24 \\
(0.45)\end{array}$ & $\begin{array}{c}0.38 \\
(0.22)\end{array}$ & $\begin{array}{c}0.17 \\
(0.59)\end{array}$ & $\begin{array}{c}0.04 \\
(0.90)\end{array}$ & $\begin{array}{c}0.52 \\
(0.08)\end{array}$ & $\begin{array}{c}0.12 \\
(0.71)\end{array}$ \\
\hline Assisting & & $\begin{array}{c}0.35 \\
(0.27)\end{array}$ & $\begin{array}{c}0.72 \\
(0.009)^{* *}\end{array}$ & $\begin{array}{c}0.66 \\
(0.02)^{*}\end{array}$ & $\begin{array}{c}0.76 \\
(0.004)^{* *}\end{array}$ & $\begin{array}{c}0.73 \\
(0.007)^{* *}\end{array}$ & $\begin{array}{c}0.76 \\
(0.004)^{* *}\end{array}$ & $\begin{array}{c}0.82 \\
(0.001)^{* *}\end{array}$ \\
\hline Define & & & $\begin{array}{c}0.33 \\
(0.29)\end{array}$ & $\begin{array}{c}0.34 \\
(0.28)\end{array}$ & $\begin{array}{c}0.29 \\
(0.37)\end{array}$ & $\begin{array}{c}0.04 \\
(0.09)\end{array}$ & $\begin{array}{c}0.56 \\
(0.06)\end{array}$ & $\begin{array}{c}0.29 \\
(0.37)\end{array}$ \\
\hline Help & & & & $\begin{array}{c}0.81 \\
(0.002)^{* *}\end{array}$ & $\begin{array}{c}0.73 \\
(0.007)^{* *}\end{array}$ & $\begin{array}{c}0.7 \\
(0.02)^{*}\end{array}$ & $\begin{array}{c}0.54 \\
(0.07)\end{array}$ & $\begin{array}{c}0.81 \\
(0.001)^{* *}\end{array}$ \\
\hline Boundaries & & & & & $\begin{array}{c}0.72 \\
(0.008)^{* *}\end{array}$ & $\begin{array}{c}0.8 \\
(0.002)^{* *}\end{array}$ & $\begin{array}{c}0.77 \\
(0.003)^{* *}\end{array}$ & $\begin{array}{c}0.82 \\
(0.001)^{* *}\end{array}$ \\
\hline Self-assured & & & & & & $\begin{array}{c}0.78 \\
(0.003)^{* *}\end{array}$ & $\begin{array}{c}0.65 \\
(0.02)^{*} \\
\end{array}$ & $\begin{array}{c}0.88 \\
(0.0001)^{* * *}\end{array}$ \\
\hline $\begin{array}{c}\text { Recovery } \\
\text { plan }\end{array}$ & & & & & & & $\begin{array}{c}0.54 \\
(0.07)\end{array}$ & $\begin{array}{c}0.82 \\
(0.001)^{* *}\end{array}$ \\
\hline Effective & & & & & & & & $\begin{array}{c}0.70 \\
(0.01)^{*}\end{array}$ \\
\hline
\end{tabular}

${ }^{a}$ : For each question, change $=$ post-training rating - pre-training rating. Pearson correlation (p-value) are reported in the table.

$* \mathrm{p}<0.05$

$* * \mathrm{p}<0.01$

$* * * \mathrm{p}<0.001$ 